\title{
A THEOREM ON APPROXIMATION OF IRRATIONAL NUMBERS BY SIMPLE CONTINUED FRACTIONS
}

\author{
by JINGCHENG TONG
}

(Received 16th April 1986)

\section{Introduction}

Let $\xi$ be an irrational number with simple continued fraction expansion $\xi=$ $\left[a_{0} ; a_{1}, a_{2}, \ldots\right], p_{n} / q_{n}$ be its $n$th convergent, $\left|\xi-p_{n} / q_{n}\right|=1 /\left(M_{n} q_{n}^{2}\right)$. The following two theorems were proved by Müller [9] and rediscovered by Bagemihl and McLaughlin [1]:

Theorem 1. For $n>1, \max \left(M_{n-1}, M_{n}, M_{n+1}\right)>\sqrt{a_{n+1}^{2}+4}$.

Theorem 2. For $n>1$, either $M_{n}>a_{n+1}+1 / a_{n+1}$ or $\min \left(M_{n-1}, M_{n+1}\right)>a_{n+1}+1 / a_{n+1}$. If $a_{n+1} \geqq 2$ in the above theorems, we have Fujiwara's theorems [4]. Since $a_{n+1} \geqq 1$, Theorem 1 implies Borel's theorem [2], and Vahlen's theorem [22] (that either $M_{n}$ or $M_{n+1}$ is greater than 2) follows from Theorem 2 . For more information, cf. $[3,5,7,10$, $12,16,17]$.

In this paper, we use the method of papers $[20,21]$ to prove a theorem, which includes Theorems 1, 2 and provides a new inequality $\max \left(M_{n-1}, M_{n+1}\right)>4 a_{n+1}\left(a_{n+1}^{2}+1\right) /$ $\left(2 a_{n+1}^{2}+1\right)$. The proof is elementary. This theorem can be used to investigate asymmetric approximation.

\section{Preliminaries}

It is well known $[5,7]$ that if $M_{n}=\left[a_{n+1} ; a_{n+2}, \ldots\right]+\left[0 ; a_{n}, a_{n-1}, \ldots, a_{1}\right]$, then $\xi-p_{n} / q_{n}=$ $(-1)^{n} /\left(M_{n} q_{n}^{2}\right),\left|\xi-p_{n} / q_{n}\right|=1 /\left(M_{n} q_{n}^{2}\right)$.

Let $P=\left[a_{n+2} ; a_{n+3}, \ldots\right] ; Q=\left[a_{n} ; a_{n-1}, \ldots, a_{1}\right]$. Then we have the following relations:

$$
\begin{gathered}
M_{n-1}=\frac{1}{a_{n+1}+P^{-1}}+Q ; \\
M_{n}=a_{n+1}+\frac{1}{P}+\frac{1}{Q} ; \\
M_{n+1}=\frac{1}{a_{n+1}+Q^{-1}}+P .
\end{gathered}
$$


We need the following two simple lemmas. They may be easily proved by evaluating the derivatives of the functions involved.

Lemma 1. Let $f(x)=1 /\left(a+x^{-1}\right)+1 /\left(r-a-x^{-1}\right)$ with $0<a<r$.

(i) The function $f(x)$ is decreasing on the interval $(1 /(r-a), 2 /(r-a))$. Thus, if $1 /(r-a)<x<2 /(r-a), f(x)>4 r /\left(r^{2}-a^{2}\right)$.

(ii) If $r \leqq 2 a$ then $f(x)$ is decreasing on the interval $(1 /(r-a)$, $\infty)$, so that if $1 /(r-a)<x$, $f(x)>r /(a(r-a))$.

Lemma 2. If $r>0$ then the function $g(x)=1 /\left(r-x^{-1}\right)+x$ is increasing on the interval $(2 / r, \infty)$. In particular, if $0<a<r$ and $x>2 /(r-a)$ then $g(x)>4 r /\left(r^{2}-a^{2}\right)$.

\section{Main results}

\section{Theorem 3.}

(i) Let $r>a_{n+1}$ be a constant. If $M_{n}<r$, then $\max \left(M_{n-1}, M_{n+1}\right)>4 r /\left(r^{2}-a_{n+1}^{2}\right)$.

(ii) Let $r$ be a constant such that $a_{n+1}<r<2 a_{n+1}$. If $M_{n}<r$, then $\min \left(M_{n-1}, M_{n+1}\right)>$ $r /\left(a_{n+1}\left(r-a_{n+1}\right)\right)$.

Proof. Since $M_{n}<r$, by (2) we have $1 / P+1 / Q=M_{n}-a_{n+1}<r-a_{n+1}$. Hence

$$
\begin{aligned}
& P>\frac{1}{r-a_{n+1}-Q^{-1}}>0 ; \\
& Q>\frac{1}{r-a_{n+1}-P^{-1}}>0 .
\end{aligned}
$$

It is easily seen that the following inequalities hold:

$$
\begin{gathered}
M_{n+1}=\frac{1}{a_{n+1}+Q^{-1}}+P>\frac{1}{a_{n+1}+Q^{-1}}+\frac{1}{r-a_{n+1}-Q^{-1}} ; \\
M_{n-1}=\frac{1}{a_{n+1}+P^{-1}}+Q>\frac{1}{r-Q^{-1}}+Q .
\end{gathered}
$$

From (4), (5), we know

$$
\begin{aligned}
& P>\frac{1}{r-a_{n+1}} ; \\
& Q>\frac{1}{r-a_{n+1}} .
\end{aligned}
$$

If $Q \leqq 2 /\left(r-a_{n+1}\right)$, by Lemma $1(i)$, (8) and (6) we have $M_{n+1}>4 r /\left(r^{2}-a_{n+1}^{2}\right)$. 
If $Q>2 /\left(r-a_{n+1}\right)$, by Lemma 2 and (7) we have $M_{n-1}>4 r /\left(r^{2}-a_{n+1}^{2}\right)$.

Therefore $\max \left(M_{n-1}, M_{n+1}\right)>4 r /\left(r^{2}-a_{n+1}^{2}\right)$.

To prove (ii), we notice that by (5)

$$
M_{n-1}=\frac{1}{a_{n+1}+P^{-1}}+Q>\frac{1}{a_{n+1}+P^{-1}}+\frac{1}{r-a_{n+1}-P^{-1}} .
$$

Since $a_{n+1}<r<2 a_{n+1}$, by Lemma 1(ii), (9), (8), (6) and (10), we have $\min \left(M_{n-1}, M_{n+1}\right)>$ $r /\left(a_{n+1}\left(r-a_{n+1}\right)\right)$.

Remark 0. If $M_{n}=r$ and $Q \neq 2 /\left(r-a_{n+1}\right)$, we still have $\max \left(M_{n-1}, M_{n+1}\right)>$ $4 r /\left(r^{2}-a_{n+1}^{2}\right)$ by the proof of Theorem 3(i).

If $M_{n}=r$ and $Q=2 /\left(r-a_{n+1}\right)$, then by (2) we have $P=2 /\left(r-a_{n+1}\right)=Q$. It is impossible because $Q$ is rational but $P$ is irrational. Therefore Theorem 3(i) can be strengthened as follows:

Theorem 3(i'), If $M_{n} \leqq r$, then $\max \left(M_{n-1}, M_{n+1}\right)>4 r\left(r^{2}-a_{n+1}^{2}\right)$.

Remark 1. Letting $r=a_{n+1}^{2}+4$ in Theorem 3(i) we have Theorem 1 .

Letting $r=a_{n+1}+1 / a_{n+1}$ in Theorem 3(ii) gives Theorem 2 and also the result that either $M_{n}>a_{n+1}+1 / a_{n+1}$ or $\max \left(M_{n-1}, M_{n+1}\right)>4 a_{n+1}\left(a_{n+1}^{2}+1\right) /\left(2 a_{n+1}^{2}+1\right)$.

The following result is a generalization of Theorem 2 .

Corollary 1. For $n>1$ and $k>0$, either $M_{n}>a_{n+1}+1 /\left(k a_{n+1}\right)$ or $\min \left(M_{n-1}, M_{n+1}\right)>$ $k a_{n+1}+1 / a_{n+1}$.

Proof. This is a special case of Theorem 3(ii) for $r=a_{n+1}+1 /\left(k a_{n+1}\right)$.

Remark 2. If $k$ is sufficiently large, comparing Theorem 2 and Corollary 1, we have an interesting conclusion: if $M_{n}$ loses a little, both $M_{n-1}$ and $M_{n+1}$ will gain a lot.

The following result, which is a corollary of Theorem 3, was obtained by Szüsz [19, Theorem 2.1].

Corollary 2. Let $\tau>0$ be a constant and $a_{n+1}>\bar{\tau}^{1}$. Then either $M_{n}>\sqrt{1+4 \tau} / \tau$ or $\max \left(M_{n-1}, M_{n+1}\right)>\sqrt{1+4 \tau}$.

Proof. If $a_{n+1} \geqq \sqrt{1+4 \tau} / \tau$, then $M_{n}>\sqrt{1+4 \tau} / \tau$ by (2). If $a_{n+1}<\sqrt{1+4 \tau} / \tau$, let $r=$ $\sqrt{1+4 \tau} / \tau$, then by Theorem $3(i)$, we have either

$$
M_{n}>\sqrt{1+4 \tau / \tau} \text { or } \max \left(M_{n-1}, M_{n+1}\right)>\frac{4 \sqrt{1+4 \tau} / \tau}{(1+4 \tau) / \tau^{2}-\tau^{-2}}=\sqrt{1+4 \tau}
$$

We give another application of Theorem 3. Kurosu [6, p. 253] proved that if $a_{n+1} \geqq 2$, then either $M_{n}>8 / 3$ or $\max \left(M_{n-1}, M_{n+1}\right)>10 / 3=3.3333$. We improve $10 / 3$ to be $24 / 7=$ 3.4285 without the restriction $a_{n+1} \geqq 2$. 
Corollary 3. Either $M_{n}>8 / 3$ or $\max \left(M_{n-1}, M_{n+1}\right)>24 / 7$.

Proof. From formula (2), we know that if $a_{n+1} \geqq 3$, then $M_{n}>3>8 / 3$. Therefore we need only consider the case $a_{n+1} \leqq 2$. Let $r=8 / 3$. By Theorem $3(\mathrm{i})$, we have either

$$
M_{n}>8 / 3 \quad \text { or } \quad \max \left(M_{n-1}, M_{n+1}\right)>\frac{4(8 / 3)}{\left(\frac{8}{3}\right)^{2}-2^{2}}=24 / 7
$$

\section{Applications to asymmetric approximation}

Segre [18] proved a theorem on asymmetric approximation, which was investigated in $[11,13,14,15,19]$. A version of LeVeque's statement [8] of Segre's theorem is the following result.

Theorem 4. Let $\tau$ be a fixed positive number. Then in the five consecutive convergents $p_{i} / q_{i}(i=n-2, n-1, n, n+1, n+2)$ of an irrational number $\xi$, at least one of them satisfies the following inequality:

$$
-\frac{1}{\sqrt{1+4 \tau} q_{i}^{2}}<\xi-\frac{p_{i}}{q_{i}}<\frac{\tau}{\sqrt{1+4 \tau} q_{i}^{2}}
$$

LeVeque [8] pointed out that in the above theorem, five convergents cannot be replaced by three. A natural question arises: when is Theorem 4 true if five is replaced by three? Using Theorem 3, we obtain some results in this connection. We first prove the following theorem, which was obtained in [8] by using Farey's series.

Theorem 5. If $n$ is an odd positive integer, then in three consecutive convergents $p_{i} / q_{i}(i=n-1, n, n+1)$ of an irrational number $\xi$, at least one satisfies (11).

Proof. Since $\xi-p_{i} / q_{i}=(-1)^{i} /\left(M_{i} q_{i}^{2}\right)$, it is easily seen that inequality (11) is equivalent to $M_{i}>\sqrt{1+4 \tau}$ if $i$ is odd, and $M_{i}>\sqrt{1+4 \tau} / \tau$ if $i$ is even.

If $a_{n+1} \geqq \sqrt{1+4 \tau}$, then $M_{n}>\sqrt{1+4 \tau}$ by formula (2); if $a_{n+1}<\sqrt{1+4 \tau}$, let $r=\sqrt{1+4 \tau}$, by Theorem 3(i) we have either

$$
M_{n}>\sqrt{1+4 \tau} \quad \text { or } \quad \max \left(M_{n-1}, M_{n+1}\right)>\frac{4 \sqrt{1+4 \tau}}{(1+4 \tau)-1^{2}}=\sqrt{1+4 \tau} / \tau .
$$

Theorem 5 is not correct for even $n$. But for some special value of $\tau$ we have an affirmative result.

Theorem 6. If $1 \leqq \tau<2+\sqrt{5}$, then at least one of the three consecutive convergents $p_{i} / q_{i}(i=n-1, n, n+1)$ of an irrational number $\xi$ satisfies inequality (11). 
Proof. We need only prove the theorem for even $n$.

Since $1 \leqq \tau<2+\sqrt{5}$, we know that $1+4 \tau-\tau^{2}>0$ and $4 \tau /\left(1+4 \tau-\tau^{2}\right) \geqq 1$. If $a_{n+1} \geqq$ $\sqrt{1+4 \tau} / \tau$, we have $M_{n}>\sqrt{1+4 \tau} / \tau$ by formula (2); if $a_{n+1}<\sqrt{1+4 \tau} / \tau$, let $r=\sqrt{1+4 \tau} / \tau$, by Theorem 3(i), we have either

$$
M_{n}>\sqrt{1+4 \tau} / \tau \quad \text { or } \quad \max \left(M_{n-1}, M_{n+1}\right)>\frac{4 \sqrt{1+4 \tau} / \tau}{(1+4 \tau) / \tau^{2}-1^{2}}=\frac{4 \tau \sqrt{1+4 \tau}}{1+4 \tau-\tau^{2}} \geqq \sqrt{1+4 \tau} .
$$

Remark 3. Letting $\tau=1$ in Theorem 6, we obtain Borel's theorem again. But if $\tau=1$ in Theorem 4, we cannot obtain Borel's theorem.

Two real numbers $\xi$ and $\xi^{\prime}$ are said to be equivalent if there are integers $a, b, c, d$ such that $\xi^{\prime}=(a \xi+b) /(c \xi+d)$ and $a d-b c= \pm 1$. If $\xi$ is not equivalent to $(\sqrt{5}+1) / 2=$ $[1 ; 1,1, \ldots]$, then for certain values of $\tau$, inequality (11) can be sharpened.

Theorem 7. If $\xi$ is an irrational number not equivalent to $(\sqrt{5}+1) / 2$, and $7 / 4<\tau<$ $(5+2 \sqrt{5}) / 4$, then there are infinitely many convergents $p_{n} / q_{n}$ satisfying the following inequality:

$$
-\frac{1}{\sqrt{1+4 \tau} q_{n}^{2}}<\xi-\frac{p_{n}}{q_{n}}<\frac{\tau-3 / 4}{\sqrt{1+4 \tau} q_{n}^{2}}<\frac{\frac{6 \sqrt{5}-10}{5}}{\sqrt{1+4 \tau} q_{n}^{2}} .
$$

Proof. Since $\xi$ is not equivalent to $(\sqrt{5}+1) / 2=[1 ; 1,1, \ldots]$, there are infinitely many $a_{n+1} \geqq 2$.

Let $n$ be odd. If $a_{n+1} \geqq \sqrt{1+4 \tau}$, then $M_{n}>\sqrt{1+4 \tau}$ by formula (2); if $a_{n+1}<\sqrt{1+4 \tau}$, let $r=\sqrt{1+4 \tau}$, by Theorem 3(i) either

$$
M_{n}>\sqrt{1+\overline{4 \tau}} \quad \text { or } \quad \max \left(M_{n-1}, M_{n+1}\right)>\frac{4 \sqrt{1+4 \tau}}{(1+4 \tau)-2^{2}}=\frac{\sqrt{1+4 \tau}}{\tau-3 / 4} .
$$

Let $n$ be even. Since $7 / 4<\tau<(5+2 \sqrt{5}) / 4$, we know that $-16 \tau^{2}+40 \tau-5>0$ and $4(4 \tau-3) /\left(-16 \tau^{2}+40 \tau-5\right) \geqq 1$. If $a_{n+1} \geqq \sqrt{1+4 \tau} /(\tau-3 / 4)$, then $M_{n}>\sqrt{1+4 \tau} /(\tau-3 / 4)$ by formula (2); if $a_{n+1}<\sqrt{1+4 \tau} /(\tau-3 / 4)$, let $r=\sqrt{1+4 \tau} /(\tau-3 / 4)$, by Theorem 3(i), we have either $M_{n}>\sqrt{1+4 \tau} /(\tau-3 / 4)$ or

$$
\max \left(M_{n-1}, M_{n+1}\right)>\frac{4 r}{r^{2}-2^{2}}=\frac{4(4 \tau-3) \sqrt{1+4 \tau}}{-16 \tau^{2}+40 \tau-5} \geqq \sqrt{1+4 \tau} .
$$

It is easily seen that

$$
\tau-3 / 4=\left(1-\frac{3}{4 \tau}\right) \tau<\left(1-\frac{3}{4(5+2 \sqrt{5}) / 4}\right) \tau=\frac{6 \sqrt{5}-10}{5} \tau .
$$

Therefore inequality (12) is correct. 
Another theorem of asymmetric approximation was given by Robinson [14]. We state it as Theorem 8 .

Theorem 8. Given any irrational number $\xi$ and any positive number $\varepsilon$, there are infinitely many rational numbers $p / q$ satisfying the following inequality:

$$
-\frac{1}{(\sqrt{5}-\varepsilon) q^{2}}<\xi-\frac{p}{q}<\frac{1}{(\sqrt{5}+1) q^{2}}
$$

For certain values of $\varepsilon$ we can show that one of any three consecutive convergents of $\xi$ satisfies inequality (13). In fact we have the following theorem.

Theorem 9. If $(20-7 \sqrt{5}) / 5<\varepsilon<\sqrt{5}-1$, then one of any three consecutive convergents of $\xi$ satisfies inequality (13).

Proof. Let $n$ be odd. Since $\varepsilon<\sqrt{5}-1$, we have $4-2 \sqrt{5} \varepsilon+\varepsilon^{2}>0$; since

$$
\varepsilon>(20-7 \sqrt{5}) / 5=0.8695>(3+\sqrt{5} \quad \sqrt{ } 10+2 \sqrt{5}) /(\sqrt{5}+1)=0.4425,
$$

we have

$$
\frac{4(\sqrt{5}-\varepsilon)}{4-2 \sqrt{5} \varepsilon+\varepsilon^{2}}>\sqrt{5}+1
$$

If $a_{n+1} \geqq \sqrt{5}-\varepsilon$, then $M_{n}>\sqrt{5}-\varepsilon$ by formula (2); if $a_{n+1}<\sqrt{5}-\varepsilon$, let $r=\sqrt{5}-\varepsilon$, by Theorem 3(i), we have either $M_{n}>\sqrt{5}-\varepsilon$ or

$$
\max \left(M_{n-1}, M_{n+1}\right)>\frac{4(\sqrt{5}-\varepsilon)}{(\sqrt{5}-\varepsilon)^{2}-1^{2}}=\frac{4(\sqrt{5}-\varepsilon)}{4-2 \sqrt{5} \varepsilon+\varepsilon^{2}}>\sqrt{5}+1 .
$$

Let $n$ be even. Since $\varepsilon \geq(20-7 \sqrt{5}) / 5$, we have $4(\sqrt{5}+1) /(5+2 \sqrt{5})>\sqrt{5}-\varepsilon$. If $a_{n+1} \geqq \sqrt{5}+1$, then $M_{n}>\sqrt{5}+1$ by formula (2); if $a_{n+1}<\sqrt{5}+1$, let $r=\sqrt{5}+1$, by Theorem 3(i), we have either

$$
M_{n}>\sqrt{5}+1 \quad \text { or } \quad \max \left(M_{n-1}, M_{n+1}\right)>\frac{4(\sqrt{5}+1)}{(\sqrt{5}+1)^{2}-1^{2}}=\frac{4(\sqrt{5}+1)}{5+2 \sqrt{5}}>\sqrt{5}-\varepsilon
$$

Therefore Theorem 9 is true.

Now two problems arise naturally.

Problem 1. Find all the values of $\tau$ such that for any irrational number $\xi$, at least one of any three consecutive convergents satisfies inequality (11). 
Problem 2. Find all the values of $\varepsilon$ such that for any irrational number $\xi$, at least one of any three consecutive convergents satisfies inequality (13).

Acknowledgement. The author thanks the referee sincerely for his valuable suggestions to improve this paper.

\section{REFERENCES}

1. F. Bagemihl and J. R. McLaughin, Generalization of some classical theorems concerning triples of consecutive convergents to simple continued fractions, J. Reine Angew. Math. 221 (1966), 146-149.

2. E. BoRel, Contribution à l'analyse arithmetique du continu, J. Math. Pures Appl. 9 (1903), 329-375.

3. J. H. E. Cohn, Hurwitz' theorem, Proc. Amer. Math. Soc. 38 (1973), 436.

4. M. Fujiwara, Bemerkung zur Theorie der Approximation der irrationalen Zahlen durch rationale Zahlen, Tohoku Math. J. 14 (1918), 109-115.

5. J. F. Koкsma, Diophantische Approximationen (Chelsea, New York, 1936).

6. K. Kurosu, Note on the theory of approximation of irrational numbers by rational numbers, Tohoku Math. J. 21 (1922), 247-260.

7. S. LANG, Introduction to Diophantine Approximations, (Addison-Wesley Publ. Co., 1966).

8. W. J. LeVeque, On asymmetric approximations, Michigan Math. J. 2 (1953), 1-6.

9. M. MÚler, Über die Approximation reeler Zahlen durch die Näherungsbruche ihres regelmässigen Kettenbruches, Arch. Math. 6 (1955), 253-258.

10. M. B. Nathanson, Approximation by continued fractions, Proc. Amer. Math. Soc. 45 (1974), 323-324. 123.

11. I. Niven, On asymmetric Diophantine approximations, Michigan Math. J. 9 (1962), 121-

12. I. Niven, Diophantine Approximations (Interscience Publishers, 1963).

13. C. D. Olds, Note on an asymmetric Diophantine approximation, Bull. Amer. Math. Soc. 52 (1946), 261-263.

14. R. M. Robinson, Unsymmetric approximation of irrational numbers, Bull. Amer. Math. Soc. 53 (1947), 351-361.

15. R. M. Robinson, The critical numbers for unsymmetric approximation, Bull. Amer. Math. Soc. 54 (1948), 693-705. 35-38.

16. A. L. Schmidt, Approximation theorems of Borel and Fujiwara, Math. Scand. 14 (1964),

17. W. Schmidt, Diophantine Approximation (Lecture Notes in Math. 785, Springer-Verlag, 1980).

18. B. Segre, Lattice points in infinite domains and asymmetric Diophantine approximation, Duke J. Math. 12 (1945), 337-365.

19. P. Szusz, On a theorem of Segre, Acta Arith. 23 (1973), 371-377.

20. J. Tong, A generalization of the Borel theorem on Diophantine approximation, Riv. Math. Univ. Parma 9 (1983), 121-124. 
21. J. Tong, The conjugate property of the Borel theorem on Diophantine approximation, Math. Z. 184 (1983), 151-153.

22. K. T. VAhlen, Über Näherungswerte und Kettenbruche, J. Reine Angew. Math. 115 (1895), 221-233.

Department of Mathematical Sciences

UNIVERSITY OF NORTH FLoRIDA

JACKSONVILLE, FL 32216

U.S.A.
Institute of Applied Mathematics

ACADEMIa Sinica

Peking

China 